\title{
Distribution of tyrosine hydroxylase-expressing interneurons with respect to anatomical organization of the neostriatum
}

\section{Bengi Ünal, Osvaldo Ibáñez-Sandoval, Fulva Shah, Elizabeth D. Abercrombie and James M. Tepper*}

Center for Molecular and Behavioral Neuroscience, Rutgers, The State University of New Jersey, Newark, NJ, USA

\section{Edited by:}

Jose Bargas, Universidad Nacional

Autónoma de México, Mexico

Reviewed by:

Abbas F. Sadikot, Mc Gill University, Canada

Lucía Prensa Sepúlveda, Universidad Autónoma de Madrid, Spain

*Correspondence:

James M. Tepper, Center for Molecular and Behavioral Neuroscience, Rutgers

University, 197 University Avenue,

Newark, NJ 07102, USA.

e-mail: jtepper@andromeda.rutgers. edu
We have recently shown in vitro that striatal tyrosine hydroxylase-expressing interneurons identified in transgenic mice by expression of enhanced green fluorescent protein (TH-eGFP) display electrophysiological profiles that are distinct from those of other striatal interneurons. Furthermore, striatalTH-eGFP interneurons show marked diversity in their electrophysiological properties and have been divided into four distinct subtypes. One question that arises from these observations is whether striatal TH-eGFP interneurons are distributed randomly, or obey some sort of organizational plan as has been shown to be the case with other striatal interneurons. An understanding of the striatal TH-eGFP interneuronal patterning is a vital step in understanding the role of these neurons in striatal functioning. Therefore, in the present set of studies the location of electrophysiologically identified striatal TH-eGFP interneurons was mapped. In addition, the distribution of TH-eGFP interneurons with respect to the striatal striosome-matrix compartmental organization was determined using $\mu$-opioid receptor (MOR) immunofluorescence or intrinsic TH-eGFP fluorescence to delineate striosome and matrix compartments. Overall, the distribution of the differentTH-eGFP interneuronal subtypes did not differ in dorsal versus ventral striatum. However, striatal TH-eGFP interneurons were found to be mostly in the matrix in the dorsal striatum whereas a significantly higher proportion of these neurons was located in MOR-enriched domains of the ventral striatum. Further, the majority of striatalTH-eGFP interneurons was found to be located within $100 \mu \mathrm{m}$ of a striosome-matrix boundary. Taken together, the current results suggest thatTH-eGFP interneurons obey different organizational principles in dorsal versus ventral striatum, and may play a role in communication between striatal striosome and matrix compartments.

Keywords: GABAergic, striatum, striosome, matrix, dopamine island

\section{INTRODUCTION}

Acquisition of motor learning and volitional initiation of behavior critically depend on faithful transfer of information among the components of the basal ganglia. The neostriatum is the largest structure and the major input nucleus of the basal ganglia. Furthermore, the striatum plays a pivotal role in the shaping of the ongoing activity of the tonically active basal ganglia nuclei, the substantia nigra pars reticulata and globus pallidus external segment, via different dopamine receptor expressing spiny projection neurons (SPNs) that give rise to the direct and indirect striatofugal pathways, respectively (Albin et al., 1989; Gerfen et al., 1990; Kawaguchi et al., 1990; Kita, 2001; Kita et al., 2006).

Spiny projection neurons account for about $95 \%$ of the striatal neuronal population (Oorschot, 2010). The remaining neurons are all local interneurons. The latter display a remarkable degree of neurochemical and electrophysiological diversity (Kawaguchi et al., 1995; Rymar et al., 2004; Oorschot, 2010). Striatal interneurons can be grouped into two main groups, cholinergic interneurons and GABAergic interneurons. The GABAergic interneurons are comprised of parvalbumin (PV)-expressing fast-spiking, neuropeptide $\mathrm{Y}(\mathrm{NPY})$-somatostatin (SOM)-nitric oxide synthase (NOS) co-expressing, calretinin (CR)-expressing (Kawaguchi, 1993; Kawaguchi et al., 1995; Wu and Parent, 2000; Tepper, 2010), and tyrosine hydroxylase (TH)-expressing (Dubach et al., 1987; Tashiro et al., 1989a; Meredith et al., 1999; Mazloom and Smith, 2006; Tande et al., 2006; Huot et al., 2007) cells.

We recently used brain slices from transgenic mice engineered to express enhanced green fluorescent protein under the control of TH-promoter (TH-eGFP) to characterize the anatomy and physiology of striatal TH neurons, and established that they are a novel class of GABAergic interneurons. We showed not only that striatal TH-eGFP neurons were markedly distinct from SPNs as well as other from other striatal interneurons but also that these neurons exhibited a striking heterogeneity in terms of their electrophysiological characteristics and could be subdivided into four distinct subtypes termed Types I-IV (Ibáñez-Sandoval et al., 2010).

Why is there such a level of electrophysiological diversity in this small group of neurons and how may it affect striatal functioning? In order to address these questions, previous findings regarding TH-eGFP interneurons should be considered within the context of the anatomical and functional heterogeneity in the striatum. The neostriatum can be subdivided into dorsal and ventral divisions, comprising the caudate nucleus and putamen, and the nucleus accumbens, respectively (Gerfen and Wilson, 1996). The nucleus accumbens can be further divided into core and shell regions, each 
with distinct inputs and outputs (Zaborszky et al., 1985; Berendse et al., 1988; Ragsdale and Graybiel, 1981; Heimer et al., 1997; Voorn et al., 2004; Ikemoto, 2007).

In addition to dorsal and ventral divisions, the striatum can be further subdivided into patch or striosome, and matrix domains, which also reflect another layer of differential afferent and efferent connectivity (Graybiel et al., 1981, 1987; Gerfen et al., 1990; Gerfen, 1992). These interdigitating compartments are neurochemically distinct. The matrix shows strong immunoreactivity for acetylcholinesterase and calbindin whereas striosomes are characterized by strong(er) expression of substance P, enkephalin, and limbic associated membrane protein (Graybiel et al., 1981; Gerfen et al., 1985; Bolam et al., 1988; Voorn et al., 1989; Holt et al., 1997). Additional striosome/matrix differences exist in terms of enhanced expression of other signaling molecules in the striosomes such as: $\mu$-opioid receptor (MOR), AMPA receptor subunit 1 (GluR1), voltage-gated potassium channel interacting protein 1 (Mikula et al., 2009), dopamine receptor subtypes (Rivera et al., 2002), and olfactory-type G-protein alpha subunit expression (Sako et al., 2010).

Although the function of this mosaic organization remains elusive, it has a critical organizing effect in striatal cytoarchitecture. The orientation and trajectory of SPN dendritic and axonal arborization obey compartmental boundaries (Kawaguchi et al., 1990), whereas processes of cholinergic and GABAergic do not (Chesselet and Graybiel, 1986; Cowan et al., 1990; Kawaguchi, 1992). Furthermore, the somata of cholinergic and NPY interneurons have been noted to be preferentially situated at the compartmental boundaries (Kubota and Kawaguchi, 1993; Martone et al., 1994; Saka et al., 2002; Bernácer et al., 2005, 2007). These data collectively suggest the existence of important differences in signal processing in striosome and matrix domains in which certain classes of striatal interneurons may play a crucial role (Saka et al., 2002).

In the current set of studies, we mapped the location of TH-eGFP cells with respect to multiple levels of the anatomical organization of the striatum. First, the locations of different electrophysiologically defined TH-eGFP subtypes within the striatal volume were determined. Second, striatal TH-eGFP neurons were mapped with respect to striosome and matrix compartments defined on the basis of MOR immunofluorescence. Finally, a small subset of TH-eGFP neurons was recorded in different compartments identified by the intensity of TH-eGFP-TH fluorescence in young mice. Our results show that electrophysiologically identified subtypes are equally prevalent in all regions of the striatum. However, when the finer level anatomical organization of the striatum is taken into account, a different pattern emerged in terms of TH-eGFP neuron distribution. In the dorsal striatum, TH-eGFP neurons are diffusely spread out with most of them residing in the matrix compartment as expected from area distribution whereas, in the ventral striatum, there is a disproportionately higher presence of TH-eGFP neurons in MOR-enriched regions.

\section{MATERIALS AND METHODS SUBJECTS}

The progeny of hemizygous $\mathrm{Tg}$ (Th-EGFP)DJ76Gsat/Mmnc (GENSAT) mice (Gong et al., 2003) obtained from the Mutant Mouse Regional Resource Centers at University of California at
Los Angeles and backcrossed to FVB mice were used in the experiments. Mice were bred and kept in the temperature and humidity controlled AAALAC-accredited animal facility and maintained on a 12/12 dark light cycle with light onset at 7 a.m. After weaning on postnatal day 21 , animals were genotyped from tail snips to confirm that they were TH-eGFP positive. All experimental protocols were in accordance with Rutgers University Institutional Animal Care and Use Committee and the NIH Guide to the Care and Use of Laboratory Animals. Utmost effort was exercised to minimize the number of mice used and the discomfort and/or pain the mice underwent.

\section{IN VITROELECTROPHYSIOLOGY}

Following intraperitoneal (i.p.) injection of ketamine $(100 \mathrm{mg} /$ kg; Ketaject, Henry Schein, Melville, NY, USA), TH-eGFP mice were transcardially perfused with ice-cold modified Ringer's solution that contained (in millimolar) 124 Choline $\mathrm{Cl}, 2.5 \mathrm{KCl}, 26$ $\mathrm{NaHCO}_{3}, 3.3 \mathrm{MgCl}_{2}, 1.2 \mathrm{NaH}_{2} \mathrm{PO}_{4}, 10$ glucose or 248 sucrose, $2.5 \mathrm{KCl}, 7 \mathrm{MgCl}_{2}, 23 \mathrm{NaHCO}_{3}, 1.2 \mathrm{NaH}_{2} \mathrm{PO}_{4}, 7$ glucose, 1 ascorbate, and 3 pyruvate. Subsequently, $250-350 \mu \mathrm{m}$ coronal or $10^{\circ}$ parahorizontal striatal sections were obtained using a vibrating microtome (Vibratome ${ }^{\mathrm{TM}} 3000$, St Louis, MO, USA). Slices were transferred initially into a slice chamber that contained Ringer's Solution (in millimolar: $124 \mathrm{NaCl}, 2.5 \mathrm{KCl}, 26 \mathrm{NaHCO}_{3}, 1.3 \mathrm{MgCl}_{2}$, $1.2 \mathrm{NaH}_{2} \mathrm{PO}_{4}, 10$ glucose, $2 \mathrm{CaCl}_{2}, 1$ ascorbic acid, 3 pyruvate, 0.4 myoinositol) maintained at $33^{\circ} \mathrm{C}$ and later kept at room temperature until the time of recording. During the recordings slices were continuously perfused with normal Ringer's solution at a flow rate of $2 \mathrm{ml} / \mathrm{min}$, which was maintained at $33^{\circ} \mathrm{C}$ via TC-324B inline heater system (Warner Instruments, Hamden, CT, USA). TH-eGFP neurons were identified by infrared DIC and epifluorescence visualization with a $40 \times$ objective using a BX50-WI Olympus microscope. Whole-cell patch clamp recordings in current clamp mode were obtained using glass pipettes (3-7 M $\Omega$ ) filled with (in millimolar): $130 \mathrm{KMeSO}_{3}, 10 \mathrm{NaCl}, 10$ HEPES, 1 EGTA, $0.1 \mathrm{CaCl}_{2}$, $2 \mathrm{MgCl}_{2}, 3$ ATP, $0.3 \mathrm{GTP}, \mathrm{pH}$ adjusted to 7.3 . Biocytin (0.2\%) was added to the intracellular solution in order to label the recorded neuron for later identification and anatomical investigation of recorded striatal TH-eGFP neurons.

Recordings were acquired with a Neurodata IR-283 current clamp amplifier and digitized at $10-40 \mathrm{kHz}$ via a Micro $1401 \mathrm{Mk}$ II data acquisition unit and transferred to a PC using Signal ${ }^{\mathrm{TM}}$ v.4 software (CED, Cambridge, UK) for offline analysis. To examine the basic membrane properties of the TH-eGFP neurons, a sequence of hyperpolarizing and depolarizing current steps from -200 to $+200 \mathrm{pA}$ were injected for $500 \mathrm{~ms}$. Current-voltage (I-V) curves were constructed by averaging $10 \mathrm{~ms}$ epochs within the last $100 \mathrm{~ms}$ of the 500-ms current injection that was devoid of action potentials (APs) or spontaneous synaptic potentials. Membrane input resistance was estimated from calculating the slope of the $\mathrm{I}-\mathrm{V}$ relation at $0 \mathrm{pA}$ current injection (resting membrane potential). AP measurements were taken from the first AP evoked by injection of threshold depolarizing current. AP half-width was determined by measuring the time between points of half-maximal AP amplitude. The spike after hyperpolarization amplitude was computed by subtracting the trough value of the 200-ms time window following the AP from the AP threshold value. Finally, the membrane time constant $(\tau)$ 
was defined as the time it takes the membrane to fall to $63 \%$ of the steady state value by fitting an exponential to the initial phase of the membrane response to a -20 pA current injection.

\section{BIOCYTIN CYTOCHEMISTRY}

Following electrophysiological characterization, sections containing biocytin-filled neurons were fixed in $4 \%$ paraformaldehyde in $0.1 \mathrm{M}$ phosphate buffer $(\mathrm{PB})$ overnight at $4^{\circ} \mathrm{C}$ and transferred next day into $0.1 \mathrm{M}$ PB. Sections were then washed for $3 \times 10 \mathrm{~min}$ in $0.1 \mathrm{M}$ PB followed by $10 \%$ methanol and $3 \% \mathrm{H}_{2} \mathrm{O}_{2}$ for $15 \mathrm{~min}$, and incubated with avidin-biotin-peroxidase complex (Vector Laboratories; $1: 200$ ) and $0.1 \%$ Triton X-100 overnight at $4{ }^{\circ} \mathrm{C}$. After washing $6 \times 10 \mathrm{~min}$ in $0.1 \mathrm{M} \mathrm{PB}$ the sections were reacted with $3,-3^{\prime}$ diaminobenzidine $(0.025 \%)$ and $\mathrm{H}_{2} \mathrm{O}_{2}(0.0008 \%)$ in $\mathrm{PB}$ with nickel intensification $(2.5 \mathrm{mM}$ nickel ammonium sulfate and $7 \mathrm{mM}$ ammonium chloride) to visualize the biocytin-stained neuron for later reconstruction using Neurolucida ${ }^{\mathrm{TM}}$ (MBF Bioscience, VT, USA). Slices were later postfixed in osmium tetroxide $(0.1 \%$ in PB) for $30 \mathrm{~min}$, dehydrated through a graded series of ethanol, followed by wash with xylene. Air-dried sections were then mounted in Depex (Electron Microscopy Sciences, PA, USA) and coverslipped.

In a subset of sections, instead of DAB, biocytin was visualized by a Texas Red streptavidin conjugate $\left(1: 200\right.$, overnight at $\left.4^{\circ} \mathrm{C}\right)$. This allowed visualization of the intrinsic fluorescent TH-eGFP signal (from somata and proximal neurites) along with detection of the biocytin fill by Texas Red.

\section{MU-OPIOID RECEPTOR IMMUNOCYTOCHEMISTRY}

Adult TH-eGFP mice were sacrificed following deep anesthesia with i.p. Ketamine $(200 \mathrm{mg} / \mathrm{kg})$. Mice were then transcardially perfused with chilled Ringer's solution followed by fixation with freshly prepared $4 \%$ paraformaldehyde added to $15 \%$ saturated picric acid in $0.1 \mathrm{M} \mathrm{PB}$. After decapitation, brains were stored in the same fixative for overnight fixation. On the next day, following several washes with PBS, $60 \mu \mathrm{m}$ free-floating sections were obtained using a Vibratome $^{\mathrm{TM}} 1200$. Sections were pretreated with $1 \%$ sodium borohydride followed by $10 \%$ methanol and $3 \% \mathrm{H}_{2} \mathrm{O}_{2}$ in $\mathrm{PBS}$ prior to incubation in $10 \%$ normal donkey serum, $2 \%$ bovine serum albumin, and $0.5 \%$ Triton X-100 for $1 \mathrm{~h}$. Next, sections were incubated in a solution containing 1:1000 rabbit anti-MOR monoclonal antibody (Immunostar Inc., Hudson, WI, USA \#24216) along with $10 \%$ normal donkey serum, $2 \%$ bovine serum albumin, and 0.5\% Triton X-100 (Sigma-Aldrich Co., St. Louis, MO, USA) in PBS for $24 \mathrm{~h}$ at room temperature. After washing three times for 10 min each in PBS, sections were transferred to a solution containing 1:500 Texas Red donkey anti-rabbit secondary (Molecular Probes, Inc., Eugene, OR, USA), 10\% normal donkey serum, and $2 \%$ bovine serum albumin in PBS at $4^{\circ} \mathrm{C}$ overnight. After three 10 min washes in PBS, sections were wet mounted in Vectashield (Vector Laboratories, Burlingame, CA, USA), coverslipped and sealed with nail polish.

\section{STEREOLOGY AND ANATOMICAL MAPPING}

For sections containing biocytin-filled neurons, the slices were outlined using Neurolucida ${ }^{\mathrm{TM}}$. Briefly, section outlines, borders of the corpus callosum, anterior commissure and striatum were traced at $10 \times$ and a marker was placed at the location of recorded
TH-eGFP somata. In order to account for variability in section condition (i.e., differential shrinkage), two sets of reference sections were cut at $100 \mu \mathrm{m}$ in the coronal or $10^{\circ}$ parahorizontal plane and Nissl-stained. Sections containing the biocytin-filled TH-eGFP somata were matched to the Nissl-stained reference sections at the closest $z$-depth and any deviations in dorso-ventral or medio-lateral aspects of the tissue were compensated for using the built-in shrinkage correction of the software. Registration to Nissl-stained reference sections thus enabled assigning individual $z$-depth values to recorded sections. A $45^{\circ}$ line spanning from the most ventral end of the lateral ventricle to the most ventral end of the external capsule was drawn to parse the striatum into dorsal and ventral divisions.

In MOR-immunolabeled sections from TH-eGFP mice, every fifth section containing the striatum was traced at $4 \times$ following random determination of the initial section by the default workflow of the Optical Fractionator probe in StereoInvestigator ${ }^{\mathrm{TM}}$ v. 9 (MBF Biosciences, Williston, VT, USA). The outlines of the entire section, the corpus callosum, the anterior commissure and the striatum were traced and visualized by different color contours. Additionally, the striatum was parsed into dorsal and ventral divisions based on the cell packing density and direction of fiber fascicles piercing the striatum, and with reference to the mouse brain atlas of Franklin and Paxinos (2001). The border between dorsal and ventral striatum was further adjusted on the basis of strong MOR staining around the nucleus accumbens core segment.

Under epifluorescence illumination at $530 \mathrm{~nm}$, patch/striosome and matrix compartments were outlined based on differential MOR immunoreactivity as it has been established that striosomes express stronger MOR-enkephalin immunoreactivity than the surrounding matrix in the dorsal striatum (Herkenham and Pert, 1981; Gerfen, 1989; Voorn et al., 1989). However, in the ventral striatum the afferent organization of MOR-rich domains resemble the dorsal striatal matrix (Jongen-Relo et al., 1993, 1994).

The methodology for stereological cell-counting procedure under epifluorescent illumination was adapted from previous reports using a similar approach (Henny and Jones, 2008; Prasad and Richfield, 2010).After virtual overlay of a $544-\mu \mathrm{m} \times 380-\mu \mathrm{m}$ grid on the contoured serial sections, cell counting was performed at $40 \times$ under epifluorescence illumination at $380 \mathrm{~nm}$ to detect striatal TH-eGFP expressing somata. At each counting site, section thickness was measured, which yielded an average tissue thickness of $50.7 \pm 1.14 \mu \mathrm{m}$ indicating that there was $\sim 16 \%$ shrinkage from the original cut thickness. Optical disector frame dimensions were set to $180 \mu \mathrm{m} \times 180 \mu \mathrm{m}$ with $30 \mu \mathrm{m} z$-depth and $5 \%$ guard zones on top and at the bottom. Only those TH-eGFP somata falling entirely within the borders, i.e., falling in between and coming in contact with inclusion borders but not touching the exclusion borders were counted. The loci of the counted TH-eGFP somata with respect to MOR intensely immunoreactive regions were also noted during counting to determine the compartmental distribution of the cells. Since section thickness was measured at every site, the numbers reported here are the stereological estimates weighed by section thickness. Raw data from biocytin-filled cells and stereologically sampled TH-eGFP neurons were aligned and overlaid using Neurolucida ${ }^{\mathrm{TM}}$ Explorer and the Solid Modeling extension module (MBF Biosciences, Williston, VT, USA). 


\section{DATA ANALYSIS}

Data were analyzed using CED Signal ${ }^{\mathrm{TM}}$, Origin 7.0, Microsoft Excel $^{\mathrm{TM}}$ and PAWS v. 18 software. Subtypes of TH-eGFP neurons were determined based on the electrophysiological criteria described in Ibáñez-Sandoval et al. (2010). For statistical comparisons, multivariate ANOVA and Bonferroni post hoc tests performed in electrophysiological recordings. Independent samples $t$-test, Pearson's chi-square test and Pearson's correlation were performed on anatomical data sets. $P$ values $<0.05$ were accepted as statistically significant.

\section{RESULTS}

\section{LOCALIZATION OF DIFFERENT TH-eGFP CELL TYPES}

Sixty-three electrophysiologically identified and biocytin-stained TH-eGFP neurons ( $n=31$ in coronal and $n=32$ in parahorizontal orientations) were mapped as shown in Figure 1. As each of the recorded slices were assigned a unique $z$-depth value on the basis of the reference sections, the data from coronal and parahorizontal slices could be merged with each other by Neurolucida Explorer. In order to obtain a combined image and data set, parahorizontally mapped TH-eGFP cells were virtually rotated $90^{\circ}$ along the anterior-posterior and $10^{\circ}$ along the dorsal-ventral axes and merged with the cluster of TH-eGFP cells obtained in the coronal orientation (Figure 1C).

Quantitative assessment of the distribution of electrophysiologically identified TH-eGFP neuron subtypes was analyzed in three dimensions (Figure 1C). In the cumulative distribution for the coronal orientation, a $45^{\circ}$ horizontal line was drawn dorsal to the anterior commissure extending from the ventral most point of the lateral ventricle to the ventral-most point of the external

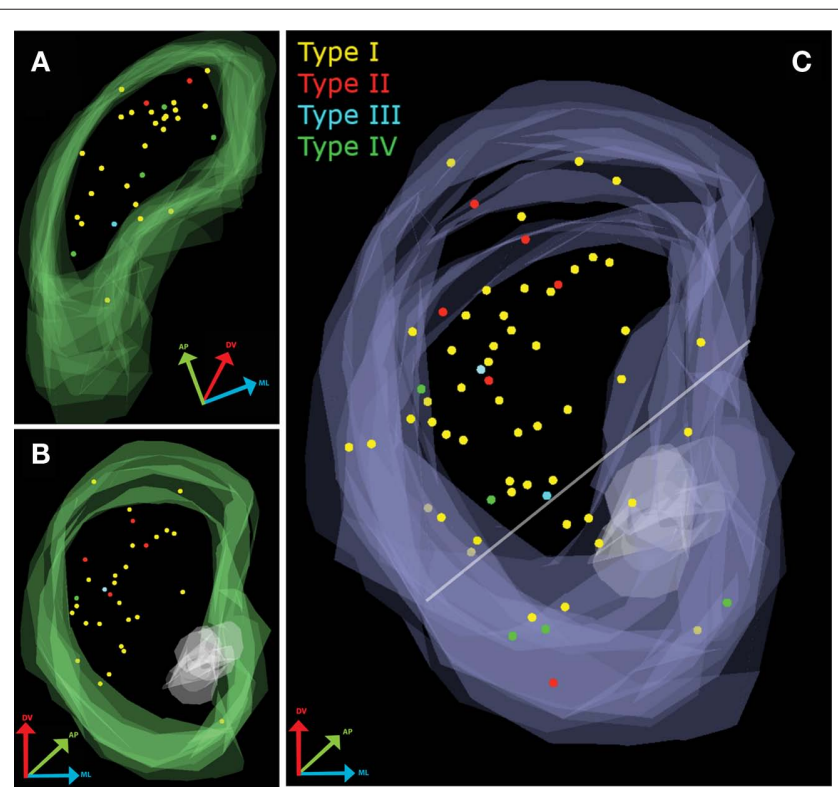

FIGURE 1 | Mapping of electrophysiologically characterized and biocytin-filledTH-EGFP neurons in the striatum. (A) Overlay of all parahorizontal sections ( $n=32)$. (B) Overlay of all coronal sections $(n=31)$. (C) Merged data set after rotating the parahorizontal sections $10^{\circ}$ from their long axis and $90^{\circ}$ around their short axis. capsule (Voorn et al., 2004) in order to mark the division between dorsal and ventral striatum. On the basis of this division, it was estimated that $81 \%(51 / 63)$ of the recorded and filled neurons were in the dorsal striatum with the remaining $19 \%$ (12/63) within the territory of the ventral striatum. Pearson's Chi-square analysis was performed in order to see whether any of the electrophysiologically distinct subtypes were disproportionately present in dorsal or ventral striatum. The results indicated that distribution of TH-eGFP interneuron subtypes did not differ in dorsal and ventral striatum [Pearson's $\left.\chi^{2}(3)=6.088, p>0.05\right]$. In both dorsal and ventral striatum, Type I TH-eGFP neurons constituted the majority of recorded neurons (Figure 1C), consistent with our previous observations (Ibáñez-Sandoval et al., 2010). Sixty percent of the Type IV neurons in our sample were located in ventral striatum but this observation did not reach statistical significance, most likely because only a few of the mapped neurons belonged to this category $(n=5)$.

\section{STEREOLOGICAL CELL COUNTING AND MAPPING WITH RELATION TO MOR STAINING DENSITY}

Striatal TH interneurons in a different set of sections were identified on the basis of their eGFP fluorescence irrespective of their electrophysiological profile and sampled using optical fractionator methodology and localized with regard to striatal MOR patches (Figures 2A-E). In the dorsal striatum, dark areas of weaker TH-eGFP fiber fluorescence were observed to show a strong overlap with intensely MOR-labeled domains (Figures 2A-C). The overall stereological estimates yielded an average of $2756 \pm 192.4$ (mean Gundersen's coefficient of error $=0.11$ ) TH-eGFP neurons per striatum per hemisphere ( $n=10$ hemispheres), in good agreement with values previously reported for striatal TH-eGFP interneurons (Ibáñez-Sandoval et al., 2010). Of the TH-eGFP neurons, $723.2 \pm 44.9$ were found to be located in MOR-enriched domains corresponding to different compartments of striatal striosomematrix organization in dorsal and ventral striatum combined. In addition, proximity to the nearest striosome-matrix boundary was measured among 332 matrix-bound TH-eGFP somata. Slightly more than half of the entire population resided within $100 \mu \mathrm{m}$ or less from the perimeter of the MOR-enriched regions in both dorsal and ventral striatum (Figure 2F).

The numbers obtained from the stereological mapping were used in regression analyses in order to understand the relationship between the TH-eGFP neurons in MOR-enriched domains and the fraction of MOR-enriched domains with respect to the entire striatum. The regression analysis indicated that the fraction of TH-eGFP neurons falling within MOR-enriched islands was dramatically different in dorsal versus ventral striatum (Figures 3A,B). Although the TH-eGFP neurons were present in MOR-enriched striosomes of the dorsal striatum at a chance level $\left(r^{2}=0.069\right.$; Figure 3C), in the ventral striatum significantly more TH-eGFP neurons were located in MOR-enriched domains than what would be expected by chance $\left(r^{2}=0.662\right.$; Figure $\left.4 \mathrm{D}\right)$. Indeed, the regression between these parameters was found to be statistically significant, as the coefficients predicting the relationship in dorsal and ventral striatum $(B=-0.332$ on dorsal striatum versus $B=0.914$ in ventral striatum) were found to differ significantly by $t$-test $[t(1)=3.935$, $p=0.001$; Figures 3C,D]. The proportion of striatal area comprised of MOR-enriched domains did not differ between dorsal 

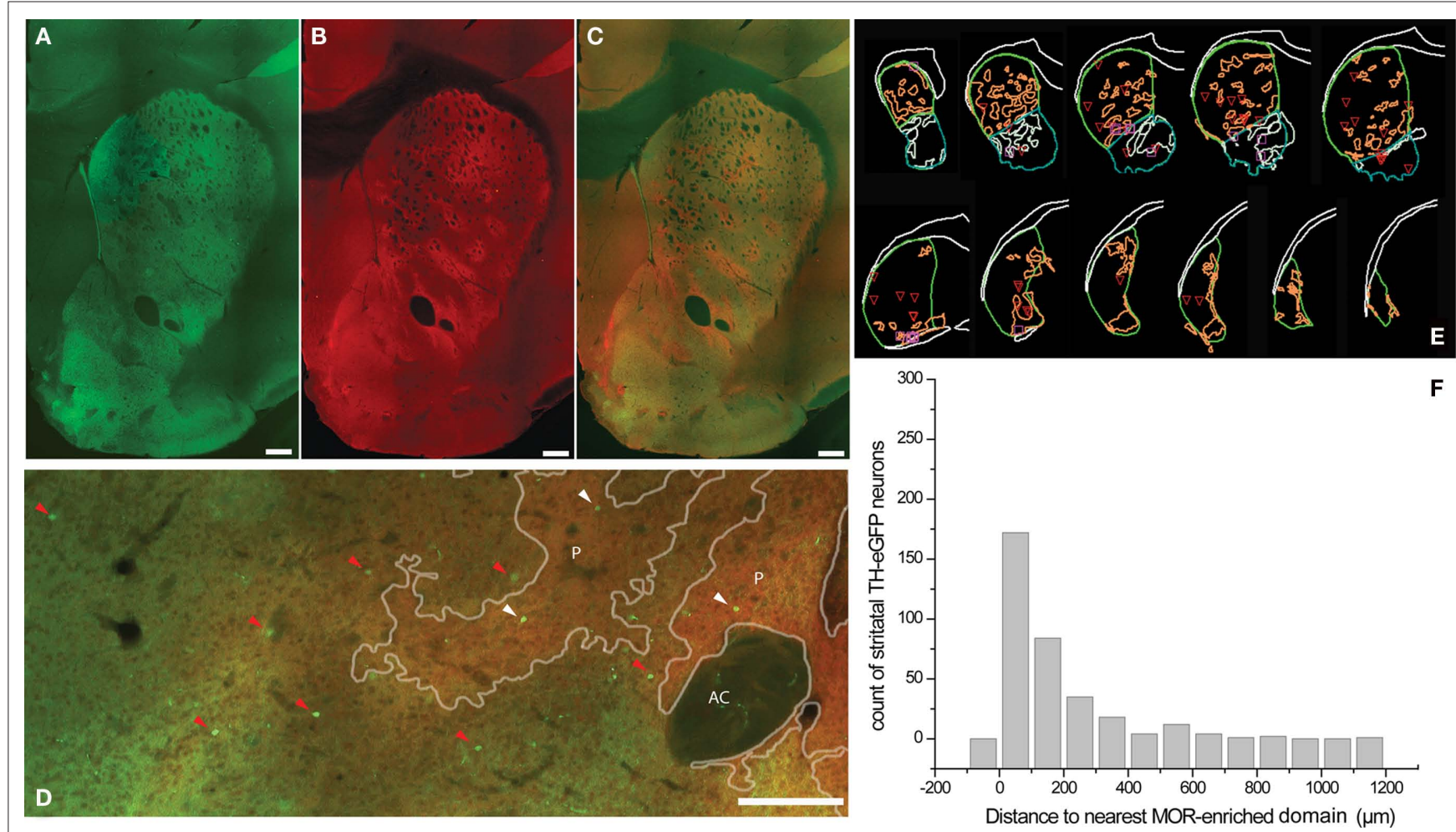

FIGURE 2 | Identification of striosome-matrix compartmentalization with MOR immunohistochemistry in TH-eGFP mice. (A-C) Typical example of a Stereolnvestigator-generated montage of a single section from anterior striatum in TH-eGFP mice showing eGFP fluorescence (A), MOR immunofluorescence (B), and the merged overlay (C). Note that the dark areas (lowerTH-eGFP fluorescence) in the dorsal striatum, show a strong overlap with the MORenriched domains (A-C). (D) Higher magnification image of a different striatal section from another animal where TH-eGFP somata were marked on the basis of their compartmental distribution. Red arrowheads point to the TH-eGFP somata in matrix, and white arrowheads point to the TH-eGFP somata in MOR-enriched striosomes (E). Representative series of $60 \mu \mathrm{m}$ sections at $300 \mu \mathrm{m}$ intervals from one hemisphere showing MOR patches. (F) The distance to the nearest MOR patch was measured among 332 TH-eGFP neurons in the matrix. The histogram shows that half of the matrix TH-eGFP neurons are located within $100 \mu \mathrm{m}$ of the striosome-matrix borders. Scale bars $=250 \mu \mathrm{m}$. $\mathrm{AC}$, anterior commissure; $\mathrm{P}, \mathrm{MOR}$ patch/striosome. and ventral striatum $[t(18)=-0.066, p=0.948]$ but interestingly in female mice ( $n=4$ hemispheres) it was found that the striatal proportion of MOR-enriched domains in ventral striatum was significantly larger than in male mice $[t(18)=-2.230, p=0.039]$. The stereological data pertaining to MOR-enriched regions are presented in Tables 1 and 2.

\section{TH-eGFP LOCATION WITH RESPECT TO TH-rich PATCHES/STRIOSOMES IN YOUNG MICE}

Ten TH-eGFP interneurons were recorded from slices taken from young (postnatal day 30-33) TH-eGFP mice where striosome and matrix compartments could be identified under epifluorescent illumination on the basis of a differential TH-fiber density. In young animals, TH-rich "islands" correspond to what will become the dopamine- and TH-poor striosome compartment in adults (Graybiel et al., 1987). Two of these neurons were located on the border between TH-rich dopamine islands and TH-poorer matrix, and both displayed the electrophysiological phenotype of Type IV TH-eGFP interneurons (Figure 4A). Interestingly, the remaining eight cells that were recorded within dopamine islands were all found to be Type I TH-eGFP interneurons (Figure 4B). The maximum projection images of the neurons in Figures $4 \mathrm{~A}, \mathrm{~B}$ taken at $5 \mathrm{~m}$ intervals indicate that in both cases, there are neurites that crossover into the neighboring compartments (Figures 4C,D). Due to the very limited size of the sample, no statistics were performed on this subset of the data.

\section{DISCUSSION \\ TH-eGFP INTERNEURONS ARE DIFFERENTIALLY DISTRIBUTED IN DORSAL AND VENTRAL STRIATUM}

The current experiments were undertaken to investigate the anatomical distribution of striatal TH-eGFP interneurons. In the first set of experiments, anatomical mapping done with electrophysiologically identified and biocytin-filled TH-eGFP neurons indicated that none of the TH-eGFP subtypes showed a dorsal-ventral or anterior-posterior patterning. In the second set, electrophysiologically unidentified TH-eGFP neurons were stereologically sampled and mapped with respect to the density of MOR immunolabeling in the striatum. The results indicate that there is a non-random distribution of TH-eGFP cells in the MOR-enriched domains in ventral striatum but not in dorsal striatum. TH-eGFP neurons were found in ventral striatal MOR-enriched domains 1.6 times more frequently than what would have expected by chance. The current results do not indicate a regional specialization of the different subtypes of TH-eGFP neurons across the dorsal-ventral divisions of the striatum. However, cortical and local stimulation 

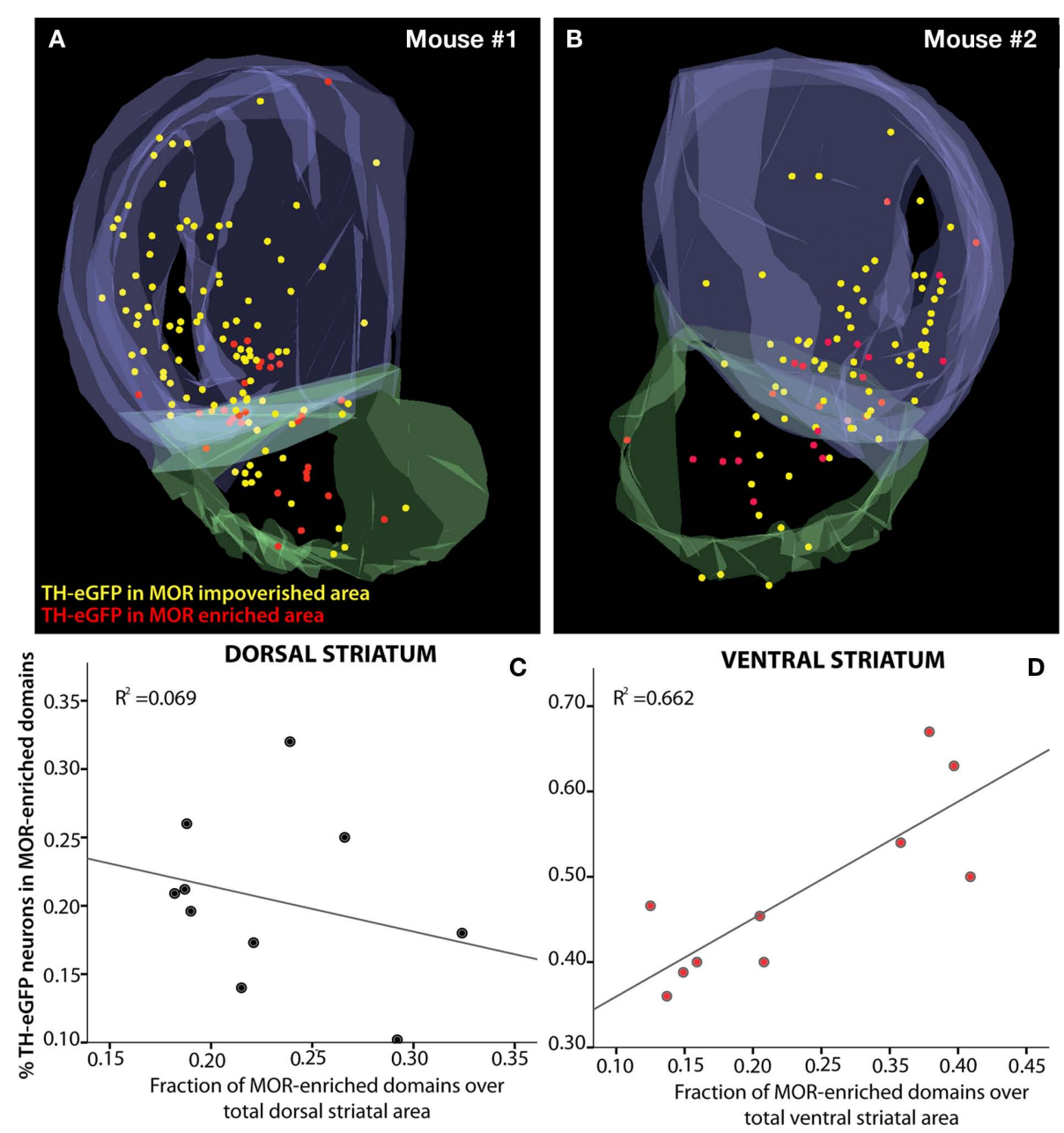

FIGURE 3 | Distribution of TH-eGFP neurons with respect to MOR-enriched areas of the striatum. The raw data ( $n=4$ hemispheres) from stereological cell-counting procedures were aligned and overlaid for 3D visualization after two hemispheres from the same brain were collapsed on each other $(\mathbf{A}, \mathbf{B})$. Markers show the distribution of systematically random sampledTH-eGFP neurons in MOR-enriched and MOR-impoverished compartments of the striatum. The plots depict the percent distribution of TH-eGFP neurons in MOR-enriched domains of dorsal and ventral striatum (C,D). The relationship between striatal MOR-enriched domains and distribution of TH-eGFP neurons with respect to these domains is significantly different in dorsal (C) and ventral (D) divisions of the striatum $(p=0.001)$. Note the differences in the scale of $y$-axes in (C,D) experiments and paired-recordings indicate that $\mathrm{TH}$ interneurons are well-integrated elements of striatal circuitry (Ibáñez-Sandoval et al., 2010). Therefore, the disparity in anatomical localization with respect to MOR-enriched domains in the dorsal and ventral striatum suggests that striatal TH interneurons may differentially influence local network dynamics in ventral versus dorsal striatum.

\section{WHY IS THE ANATOMICAL DISTRIBUTION OF TH-EGFP INTERNEURONS DIFFERENT IN THE DORSAL AND VENTRAL STRIATUM?}

And further, what is the significance of these differences in anatomical localization? The clues to the answers may lie within the rules that govern the developmental organization of the dorsal and ventral striatal divisions. It has been shown that striatal SPNs and PV-expressing interneurons follow different timelines for embryonic maximal birthrate and postmitotic maturation in dorsal striatum versus nucleus accumbens (Sadikot and Sasseville, 1997). Furthermore, van Vulpen and van der Kooy (1998) found a curious relationship between birthdates and compartmental localization of cholinergic interneurons: the earliest born cholinergic neurons were more likely to be situated in the striosome compartment whereas later born cholinergic neurons had a higher likelihood to wind up in the matrix. It is possible that ventral striatal TH-eGFP neurons have earlier birthdates than dorsal striatal ones, which may account for different compartmental localization of these neurons across the dorsal-ventral axis of the striatum. Currently, the only available findings from a developmental stance come from the early postnatal maturation of striatal $\mathrm{TH}$ interneurons (Busceti et al., 2008). Further experiments are needed to examine the embryonic development and anatomical migratory patterns of TH-eGFP neurons.

Apart from the possibility that there is difference in birthdates, one also must take into account the fact that compartmental organization reflects a complex molecular patterning requiring the orchestrated action of multiple molecules including retinoic 

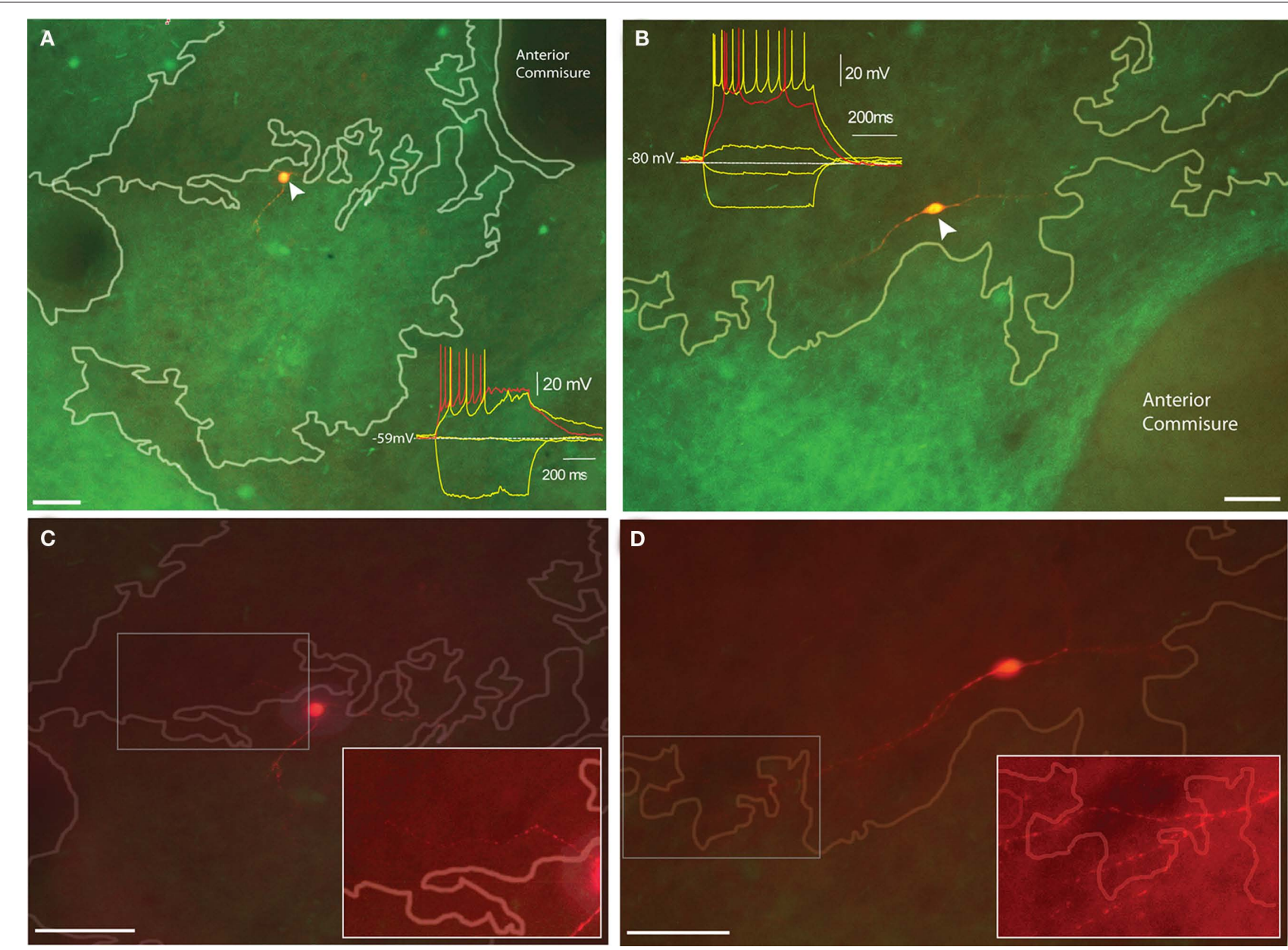

FIGURE 4 | Distribution of electrophysiologically identifiedTH-eGFP neurons with respect to dopamine islands in juvenileTH-eGFP mice. (A) Biocytin-filled Type ITH-eGFP interneuron in a dopamine island. Inset shows the I-V plot that identified the neuron as a Type I. (B) Biocytin-filled Type IVTH-eGFP interneuron with the inset showing the $I-V$ plot that identified the subtype. (C) The maximal projection of an image stack obtained at $5 \mu \mathrm{m}$ intervals of the Type I
TH-eGFP neuron in (A). The boxed region corresponds to the contrast-enhanced inset showing the proximal neurite crossing a compartmental boundary. (D) The maximal projection of the image stack obtained at $5 \mu \mathrm{m}$ intervals of the Type IV TH-eGFP neuron in (B). The boxed region corresponds to the contrast-enhanced inset showing the distal neurites crossing a compartmental boundary. Scale bars, $50 \mu \mathrm{m}$. Note different scale markers in [(A,C), (B,D)].

Table 1 | Stereological estimates of the number of TH-eGFP neurons from $60 \mu \mathrm{m}$ serial sections at $300 \mu \mathrm{m}$ intervals that were used for stereological counting and mapping of TH-eGFP neurons with respect to MOR-labeling.

Stereological estimates of striatalTH-eGFP neurons/hemisphere (mean \pm SEM)

Total number of striatalTH-eGFP neurons outside MOR-enriched domains

Total number of striatalTH-eGFP neurons inside MOR-enriched domains

Total striatalTH-eGFP neurons

Total number of dorsal striatal TH-eGFP outside MOR-enriched domains

Total number of dorsal striatal TH-eGFP neurons inside MOR-enriched domains

Total number of ventral striatal TH-eGFP neurons outside MOR-enriched domains

Total number of ventral striatal TH-eGFP neurons inside MOR-enriched domains
$2032.8 \pm 226.7$

$723.2 \pm 44.9$

$2756 \pm 192.4$

$1663.6 \pm 115.1$

$422.1 \pm 55.4$

$369.2 \pm 47.3$

$301.1 \pm 19.5$ acid (Liao et al., 2008), notch signaling (Mason et al., 2005), certain transcription factors (Arlotta et al., 2008) cell adhesion (Redies et al., 2002) and guidance molecules (Janis et al., 1999; Hamasaki et al., 2001). Differential sensitivity to these factors in dorsal and ventral striatum may have given rise to the differences in compartmental localization of TH-eGFP interneurons. In particular, it has been shown that the repulsive action of netrins is integral for proper migration of matrix-bound neuronal precursors 
Table 2 | Cavalieri estimations of surface area measurements obtained from $60 \mu \mathrm{m}$ serial sections at $300 \mu \mathrm{m}$ intervals that were used for stereological counting and mapping of TH-eGFP neurons with respect to MOR-labeling.

\begin{tabular}{|c|c|c|}
\hline \multirow{2}{*}{ Region } & \multicolumn{2}{|c|}{$\begin{array}{c}\text { Surface area } \\
\left(\mu \mathrm{m}^{2}\right) / \text { hemisphere }\end{array}$} \\
\hline & $\begin{array}{l}\text { Males ( } n=6 \\
\text { hemispheres) }\end{array}$ & $\begin{array}{l}\text { Females ( } n=4 \\
\text { hemispheres) }\end{array}$ \\
\hline Total striatal area (A) & 39058008 & 41054280 \\
\hline $\begin{array}{l}\text { Total area of MOR-enriched } \\
\text { domains (B) }\end{array}$ & 8800958 & 9404603 \\
\hline$(B) /(A)$ & $22.5 \%$ & $22.9 \%$ \\
\hline Total dorsal striatal area (C) & 28785228 & 29457135 \\
\hline $\begin{array}{l}\text { Total area of dorsal MOR-enriched } \\
\text { domains (D) }\end{array}$ & 7084028 & 6145060 \\
\hline$(\mathrm{D}) /(\mathrm{C})$ & $24.6 \%$ & $20.9 \%$ \\
\hline Total ventral striatal area (E) & 10272779 & 11597145 \\
\hline $\begin{array}{l}\text { Total area of ventral MOR-enriched } \\
\text { domains (F) }\end{array}$ & $1716929 * *$ & $4207880^{* *}$ \\
\hline$(F) /(E)$ & $16.7 \% * *$ & $36.3 \% * *$ \\
\hline
\end{tabular}

The independent t-test comparisons only indicated a significant difference for MOR-enriched domain estimations in the ventral striatum between the sexes. * indicates, $p<0.01$.

(Hamasaki et al., 2001). Subsequently, it was demonstrated that netrin-1 shows a pronounced ventral to dorsal gradient, and that cholinergic interneurons co-expressing netrin-1 largely fall within the ventral striatum (Schatzmiller et al., 2008). The same study also found that SOM-expressing GABAergic interneurons co-localized netrin-1 whereas PV and CR-expressing GABAergic interneurons did not. Since PV and CR interneurons do not show compartmental preferences within the striatum (Cowan et al., 1990; Rymar et al., 2004) and cholinergic and NPY-SOM neurons respect compartmental boundaries (Kubota and Kawaguchi, 1993; Martone et al., 1994; Saka et al., 2002), the possibility arises that expression of netrins and/or netrin receptors by TH-eGFP interneurons may be a determining factor of the compartmental localization in the dorsal and ventral striatum.

Although the current results show that there is a disproportionately higher number of TH-eGFP interneurons in MOR-enriched domains of the ventral striatum $(\sim 40$ versus $\sim 25 \%$ in the dorsal striatum), the majority of the $\mathrm{TH}$ interneurons reside in MORimpoverished areas. A closer examination of matrix TH-eGFP neurons indicated that half of the population dwells within $100 \mu \mathrm{m}$ or less of the striosome-matrix borders. This is consistent with results reported by Busceti et al. (2008) in which the majority of striatal TH neurons clustered near the pioneering nigral dopamine fibers commonly referred as "dopamine islands," which are destined to form striatal striosomes in the adult (Graybiel et al., 1981; Miura et al., 2007, 2008).

These data suggest that even if they do not reside in striosomes, striatal TH neurons may still participate in intercompartmental communication in a manner similar to that of cholinergic and NPY interneurons (Kawaguchi, 1992; Kubota and Kawaguchi, 1993; Saka et al., 2002). In order to better understand such a functional role full reconstructions of axonal and dendritic arborization of biocytin-filled TH-eGFP neurons with respect to striosome and matrix compartments in mature mice are necessary.

\section{SUBTYPE-SPECIFIC LOCALIZATION OF TH INTERNEURONS TO TH-eGFP PATCHES/STRIOSOMES IN JUVENILE STRIATUM}

In terms of the overall anatomical distribution of electrophysiological subtypes of TH-eGFP interneurons, no preferential regional localization was observed. However, one must bear in mind that there were only a few examples from some electrophysiological subtypes available for anatomical mapping. With an increased number of cases representing each TH-eGFP interneuronal subtype, a clearer picture of any preferential anatomical distribution might be revealed. Still, the preliminary results in this paper in which electrophysiological characterization of TH-eGFP neurons was carried out along with simultaneous compartmental localization based on TH-fiber intensity in young TH-eGFP mice ( $n=10$ neurons) suggest that there is chance that subtypes are localized in different compartments. It is interesting that 8 of 10 such labeled neurons in TH-rich islands displayed characteristics of Type I neurons, the most abundant electrophysiological subtype ( $>50 \%$ of entire recorded population, Ibáñez-Sandoval et al., 2010), whereas the remaining two somata that were located in the TH-poor zones displayed the phenotype of Type IV neurons, the second most prevalent subtype ( $25 \%$, Ibáñez-Sandoval et al., 2010). We would have expected to see just the opposite - the more abundant phenotype present in the more abundant striatal matrix compartment - if indeed TH-fiber intense zones correspond to striosomes as suggested (Miura et al., 2007, 2008). The mice from which these recordings and biocytin labeling obtained were aged between PN30-P33, and it is possible that this is a developmental range in which differences in TH-fiber density could be detected but compartments had already started maturation. Thus, TH-fiber rich zones in PN30-33 might correspond to matrix whereas TH-poor(er) zones might have been the striosomes. Indeed, it has been documented in adult cat, monkey and human brains that striosomes show reduced $\mathrm{TH}$ immunoreactivity (Graybiel et al., 1987). Indeed, in the MOR-immunolabeled striatal sections from adult TH-eGFP mice ( $>3$ months), we have noticed that the dorsal striatal zones showing an attenuated background TH-eGFP fiber density showed a consistent overlap with the MOR-enriched domains (Figure 2A).

Therefore, it can be argued that depending on the degree of postnatal maturation; TH-fiber-enriched domains in TH-eGFP mice may correspond to patches/striosomes or matrix. However, in either case - juvenile or fully mature-, intensity of the TH-eGFP fiber background can be a distinguishing feature of striatal compartments in these mice. It is interesting to note that at least for Type I and Type IV neurons, striatal TH-eGFP interneurons have processes that cross the boundaries between the TH-enriched regions and the rest of the striatum. To the extent that the $\mathrm{TH}$-enriched regions are in fact the precursors to the adult striosomes, this finding indicates that the axons and dendrites of striatal TH interneurons mediate intercompartment communication. 


\section{TH-eGFP INTERNEURONS, THE STRIOSOME-MATRIX MOSAIC AND STRIATAL FUNCTION}

Although the exact significance of the mosaic organization of striatum is poorly understood, there is evidence that each compartment may be involved in different aspects of striatal functioning. For instance, the matrix was found to show higher metabolic responses as a result of unrestrained activity, somatosensory stimulation, and brief restraint (Brown et al., 2002). In addition, concurrent dopamine receptor stimulation, cocaine/apomorphine induced stereotypy, and behavioral sensitization lead to differential activation of striatal compartments as measured in terms of early immediate gene responses (Saka et al., 2002). Furthermore, it has been shown by targeted ablation of NPY$\mathrm{SOM}^{+}$and cholinergic populations using a saporin toxin conjugate against a tachykinin receptor subtype that these interneurons are essential for compartmentally distinct patterns of activation to emerge in the striatum (Saka et al., 2002). Therefore, striatal TH interneurons may be working in parallel with NPY-SOM and cholinergic interneurons in the establishment of regionally different striatal activity patterns. Further anatomical and electrophysiological studies are imperative to determine whether there is a special relationship among these interneurons across striatal compartments.

\section{CONCLUSION}

Above all, the current results should motivate further inquiries regarding the precise neurochemical makeup and function of the striatal TH-expressing neurons. There is still controversy concerning the neurochemical heterogeneity in this population. On the one hand, there is strong evidence that they express GABAergic markers and exert GABA-mediated inhibition onto SPNs (Dubach et al., 1987; Cosette et al., 2005b; Mazloom and Smith, 2006; Huot and Parent, 2007; Ibáñez-Sandoval et al., 2010). In addition, there are indications that these cells may possess the cellular components

\section{REFERENCES}

Albin, R. L., Young, A. B., and Penney, J. B. (1989). The functional anatomy of basal ganglia disorders. Trends Neurosci. 12, 366-375.

Arlotta, P., Molyneaux, B. J., Jabaudon, D., Yoshida, Y., and Macklis, J. D. (2008). Ctip2 controls the differentiation of medium spiny neurons and the establishment of the cellular architecture of the striatum. J. Neurosci. 28, 622-632.

Berendse, H. W., Voorn, P., te Kortschot, A., and Groenewegen, H. J. (1988). Nuclear origin of thalamic afferents of the ventral striatum determines their relation to patch/matrix configurations in enkephalin-immunoreactivity in the rat. J. Chem. Neuroanat. 1,3-10.

Bernácer, J., Prensa, L., and GiménezAmaya, J. M. (2005). Morphological features, distribution and compartmental organization of the nicotinamide adenine dinucleotide phosphate reduced-diaphorase interneurons in the human striatum. J. Comp. Neurol. 489, 311-327.
Bernácer, J., Prensa, L., and GiménezAmaya, J. M. (2007). Cholinergic interneurons are differentially distributed in the human striatum. PLoS ONE 2, e1174. doi: 10.1371/journal. pone. 0001174

Betarbet, R., Turner, R., Chockkan, V., DeLong, M. R., Allers, K. A., Walters, J., Levey, A. I., and Greenamyre, J. T. (1997). Dopaminergic neurons intrinsic to the primate striatum. J. Neurosci. 17, 6761-6768.

Bolam, J. P., Izzo, P. N., and Graybiel, A. M. (1988). Cellular substrate of the histochemically defined striosome/matrix system of the caudate nucleus: a combined Golgi and immunocytochemical study in cat and ferret. Neuroscience 24, 853-875.

Brown, L. L., Feldman, S. M., Smith, D. M., Cavanaugh, J. R., Ackermann, R. F., and Graybiel, A. M. (2002). Differential metabolic activity in the striosome and matrix compartments of the rat striatum during natural behaviors. J. Neurosci. 22, 305-314.

necessary to synthesize, store, and release dopamine (Tashiro et al., 1989b; Mura et al., 1995, 2000; Betarbet et al., 1997; Lopez-Real et al., 2003; Cosette et al., 2005a; Tande et al., 2006; Busceti et al., 2008). The question, in the light of the current findings, is whether anatomically distinct localized and/or electrophysiologically different TH-eGFP cells are neurochemically the same or dissimilar. The answer is important because it may implicate a role for the TH-eGFP neurons in the observed spatial and temporal heterogeneity of striatal dopamine dynamics (Rodriguez et al., 2006; Wightman et al., 2007).

Finally, the current anatomical results will be essential to interpret commonly reported changes in striatal TH-expressing neuron number following experimentally induced or disease-related loss of striatal dopamine (Dubach et al., 1987; Tashiro et al., 1989a,b; Betarbet et al., 1997; Meredith et al., 1999; Mao et al., 2001; Palfi et al., 2002; Jollivet et al., 2004; Mazloom and Smith, 2006; Tande et al., 2006). Whether the appearance of "novel" TH neurons as a result of dopamine loss follows a distinct anatomical pattern would be very interesting to uncover, as striatal compartments were shown to display separable degrees of susceptibility to toxins and disease-related degeneration (Lawhorn et al., 2008; Sato et al., 2008; Granado et al., 2010). The present findings indicate that TH-eGFP neurons follow a non-random, distinct pattern of distribution across striosome-matrix compartments throughout the dorsal-ventral axis of the striatum. These results provide further impetus for future studies as to whether and how TH-eGFP neurons may affect striatal information processing based their anatomical location.

\section{ACKNOWLEDGMENTS}

The authors thank Dr. Laszlo Zaborszky for his critical suggestions on anatomical mapping methodology. This work was supported by NS034865 to James M. Tepper, NS059921 to Elizabeth D. Abercrombie and Rutgers University.

Busceti, C. L., Biagioni, F., Mastroiacovo, F., Bucci, D., Lenzi, P., Pasquali, L., Trabucco, A., Nicoletti, F., and Fornai, F. (2008). High number of striatal dopaminergic neurons during early postnatal development: correlation analysis with dopaminergic fibers. J. Neural Trans. 115, 1375-1383.

Chesselet, M. F., and Graybiel, A. M. (1986). Striatal neurons expressing somatostatin-like immunoreactivity: evidence for a peptidergic interneuronal system in the cat. Neuroscience 17, 547-571.

Cosette, M., Levesque, D., and Parent, A. (2005a). Neurochemical characterization of dopaminergic neurons in the human striatum. Parkinsonism Relat. Disord. 11, 277-286.

Cosette, M., Lecomte, F., and Parent, A. (2005b). Morphology and distribution of dopaminergic neurons intrinsic to the human striatum. J. Chem. Neuroanat. 29, 1-11.

Cowan, R. L., Wilson, C. J., Emson, P. C., and Heizmann, C. W. (1990).
Parvalbumin-containing GABAergic interneurons in the rat neostriatum. J. Comp. Neurol. 302, 197-205.

Dubach, M., Schmidt, R., Kunkel, D., Bowden, D. M., Martin, R., and German, D. C. (1987). Primate neostriatal neurons containing tyrosine hydroxylase: immunohistochemical evidence. Neurosci. Lett. 75, 205-210.

Franklin, K. B. J., and Paxinos, G. (2001). The Mouse Brain in Stereotaxic Coordinates. San Diego, CA: Academic Press.

Gerfen, C. R. (1989). The neostriatal mosaic: striatal patch-matrix organization is related to cortical lamination. Science 246, 385-388.

Gerfen, C. R. (1992). The neostriatal mosaic: multiple levels of compartmental organization. Trends Neurosci. 15, 133-139.

Gerfen, C. R., Baimbridge, K. G., and Miller, J. J. (1985). The neostriatal mosaic: compartmental distribution of calcium-binding protein and 
parvalbumin in the basal ganglia of the rat and monkey. Proc. Natl. Acad. Sci. U.S.A. 82, 8780-8784.

Gerfen, C. R., Engber, T. M., Mahan, L. C., Susel, Z., Chase, T. N., Monsma, F. J. Jr., and Sibley, D. R. (1990). D1 and $\mathrm{D} 2$ dopamine receptor-regulated gene expression of striatonigral and striatopallidal neurons. Science 250, 1429-1432.

Gerfen, C. R., and Wilson, C. J. (1996). "The basal ganglia," in Handbook of Chemical Neuroanatomy, Vol. 12, Integrated Systems of the CNS, Part III, eds L. W. Swanson, A. Björklund, and T. Hökfelt (Amsterdam: Elsevier), 371-468.

Gong, S., Zheng, C., Doughty, M. L., Losos, K., Didkovsky, N., Schambra, U. B., Nowak, N. J., Joyner, A., Leblanc, G., Hatten, M. E., and Heintz, N. (2003). A gene expression atlas of the central nervous system based on bacterial artificial chromosomes. Nature 425 , 917-925.

Granado, N., Ares-Santos, S., O'Shea, E., Vicario-Abejon, C., Colado, M. I., and Moratalla, R. (2010). Selective vulnerability in striosomes and in the nigrostriatal dopaminergic pathway after methamphetamine administration: early loss of TH in striosomes after methamphetamine. Neurotox. Res. 18, 48-58.

Graybiel, A. M., Hirsch, E. C., and Agid, Y. A. (1987). Differences in tyrosine hydroxylase-like immunoreactivity characterize the mesostriatal innervation of striosomes and extrastriosomal matrix at maturity. Proc. Natl. Acad. Sci. U.S.A. 84, 303-307.

Graybiel, A. M., Pickel, V. M., Joh, T. H., Reis, D. J., and Ragsdale, C. W. (1981). Direct demonstration of a correspondence between the dopamine islands and acetylcholinesterase patches in the developing striatum. Proc. Natl. Acad. Sci. U.S.A. 78, 5871-5875.

Hamasaki, T., Goto, S., Nishikawa, S., and Ushio, Y. (2001). A role of netrin-1 in the formation of subcortical structure striatum: repulsive action on the migration of late-born striatal neurons. J. Neurosci. 21, 4272-4280.

Heimer, L., Alheid, G. F., de Olmos, J. S., Groenewegen, H. J., Haber, S. N., Harlan, R. E., and Zahm, D. S. (1997). The accumbens: beyond the core-shell dichotomy. J. Neuropsychiatry Clin. Neurosci. 9, 354-381.

Henny, F., and Jones, B. E. (2008). Projections from basal forebrain to prefrontal cortex comprise cholinergic, GABAergic and glutamatergic inputs to pyramidal cells or interneurons. Eur. J. Neurosci. 27, 654-670.

Herkenham, M., and Pert, C. B. (1981). Mosaic distribution of opiate recep- tors, parafascicular projections and acetylcholinesterase in the striatum. Nature 291, 415-418.

Holt, D. J., Graybiel, A. M., and Saper, C. B. (1997). Neurochemical architecture of the human striatum. J. Comp. Neurol. 384, 1-25.

Huot, P., Levesque, M., and Parent, A. (2007). The fate of striatal dopaminergic neurons in Parkinson's disease and Huntington's chorea. Brain 130, 222-232.

Huot, P., and Parent, A. (2007). Dopaminergic neurons intrinsic to the striatum. J. Neurochem. 101, 1441-1447.

Ibáñez-Sandoval, O., Tecuapetla, F., Unal, B., Shah, F., Koós, T., and Tepper, J. M. (2010). Electrophysiological and morphological characteristics and synaptic connectivity of tyrosine hydroxylase-expressing neurons in adult mouse striatum. J. Neurosci. 30, 6999-7016.

Ikemoto, S. (2007). Dopamine rewardcircuitry: two projection systems from the ventral midbrain to the nucleus accumbens-olfactory tubercle complex. Brain Res. Rev. 56, 27-78.

Janis, L. S., Cassidy, R. M., and Kromer, L. F. (1999). Ephrin-A binding and EphA receptor expression delineate the matrix compartment of the striatum. J. Neurosci. 19, 4962-4971.

Jollivet, C., Montero-Menei, C. N., Venier-Julienne, M. C., Sapin, A., Benoit, J. P., and Menei, P. (2004). Striatal tyrosine hydroxylase immunoreactive neurons are induced by L-dihydroxyphenylalanine and nerve growth factor treatment in 6-hydroxydopamine lesioned rats. Neurosci. Lett. 362, 79-82.

Jongen-Relo, A., Groenewegen, H. J., and Voorn, P. (1993). Evidence for multicompartmental histochemical organization of the nucleus accumbens in the rat. J. Comp. Neurol. 337, 267-276.

Jongen-Relo, A. L., Voorn, P., and Groenewegen, H. J. (1994). Immunohistochemical characterization of the shell and core territories of the nucleus accumbens in the rat. Eur. J. Neurosci. 6, 1255-1264.

Kawaguchi, Y. (1992). Large aspiny cells in the matrix of the rat neostriatum in vitro: physiological identification, relation to the compartments and excitatory postsynaptic currents. $J$. Neurophysiol. 67, 1669-1682.

Kawaguchi, Y. (1993). Physiological, morphological and histochemical characterization of three classes of interneurons in the neostriatum. J. Neurosci. 13, 4908-4923.

Kawaguchi, Y., Wilson, C. J., Augood, S. J., and Emson, P. C. (1995). Striatal interneurones: chemical, physiological and morphological characterization. Trends Neurosci. 18, 527-535.

Kawaguchi, Y., Wilson, C. J., and Emson, P. C. (1990). Projection subtypes of rat neostriatal matrix cells revealed by intracellular injection of biocytin. J. Neurosci. 10, 3421-3438.

Kita, H. (2001). Neostriatal and globus pallidus stimulation induced inhibitory postsynaptic potentials in entopeduncular neurons in rat brain slice preparations. Neuroscience 105, 871-879.

Kita, H., Chiken, S., Tachibana, Y., and Nambu, A. (2006). Origins of GABA(A) and GABA(B) receptormediated responses of the globus pallidus induced after stimulation of the putamen in the monkey. J. Neurosci. 26, 6554-6562.

Kubota, Y., and Kawaguchi, Y. (1993). Spatial distributions of chemically identified intrinsic neurons in relation to patch and matrix compartments of rat neostriatum. J. Comp. Neurol. 332, 499-513.

Lawhorn, C., Smith, D. M., and Brown, L. L. (2008). Striosome-matrix pathology and motor deficits in the YAC 128 mouse model of Huntington's disease. Neurobiol. Dis. 32, 471-478.

Liao, L., Tsai, H., Wang, H., Chang, J., Lu, $\mathrm{K}$., Wu, H., and Lee, Y. (2008). Modular patterning of structure and function of the striatum by retinoid receptor signaling. Proc. Natl. Acad. Sci. U.S.A. 105, 6765-6770.

Lopez-Real, A., Rodriguez-Pallares, J., Guerra, M. H., and LabandeiraGarcia, J. L. (2003). Localization and functional significance of striatal neurons immunoreactive to aromatic L-amino acid decarboxylase or tyrosine hydroxylase in rat parkinsonian models. Brain Res. 969, 135-146.

Mao, L., Lau, Y.S., Petroske, E., and Wang, J. Q. (2001). Profound astrogenesis in the striatum of adult mice following nigrostriatal dopaminergic lesion by repeated MPTP administration. Brain Res. Dev. Brain Res. 131, 57-65.

Martone, M. E., Young, S. J., Armstrong, D. M., and Groves, P. M. (1994). The distribution of cholinergic perikarya with respect to enkephalin-rich patches in the caudate nucleus of the adult cat. $J$. Chem. Neuroanat. 8, 47-59.

Mason, H. A., Rakowiecki, S. M., Raftopoulou, M., Nery, S., Huang, Y., Gridley, T., and Fishell, G. (2005). Notch signaling coordinates the pattern of striatal compartments. Development 132, 4247-4258.

Mazloom, M., and Smith, Y. (2006). Synaptic microcircuitry of tyrosine hydroxylase-containing neurons and terminals in the striatum of 1-Methyl4-Phenyl-1,2,3,6-Tetrahydropyridine treated monkeys. J. Comp. Neurol.495, 453-469.

Meredith, G. E., Farrell, T., Kellaghan, P., Tan, Y., Zahm, D. S., and Toterdell, S. (1999). Immunocytochemical characterization of catecholaminergic neurons in the rat striatum following dopamine-depleting lesions. Eur. J. Neurosci. 11, 3583-3596.

Mikula, S., Parrish, S. K., Trimmer, J. S., and Jones, E. G. (2009). Complete 3D visualization of primate striosomes by KChIP1 immunostaining. J. Comp. Neurol. 514, 507-517.

Miura, M., Masada, M., and Aosaki, T. (2008). Roles of micro-opioid receptors in GABAergic synaptic transmission in the striosome and matrix compartments of the striatum. Mol. Neurobiol. 37, 104-115.

Miura, M., Saino-Saito, S., Masuda, M., Kobayashi, K., and Aosaki, T. (2007). Compartment-specific modulation of GABAergic synaptic transmission by $\mu$-opioid receptor in the mouse striatum with green fluorescent protein-expressing dopamine islands. J. Neurosci. 27, 9721-9728,

Mura, A., Jackson, D., Manley, M. S., Young, S. J., and Groves, P. M. (1995). Aromatic L-amino acid decarboxylase immunoreactive cells in the rat striatum: a possible site for the conversion of exogenous L-DOPA to dopamine. Brain Res. 704, 51-60.

Mura, A., Linder, J. C., Young, S. J., and Groves, P. M. (2000). Striatal cells containing aromatic L-amino acid decarboxylase: an immunohistochemical comparison with other classes of striatal neurons. Neuroscience 98 , 501-511.

Oorschot, D. E. (2010). "Cell types in the different nuclei of the basal ganglia," in Handbook of Basal Ganglia Structure and Function, eds H. Steiner and K. Y. Tseng (San Diego, CA: Academic Press), 63-91.

Palfi, S., Leventhal, L., Chu, Y., Ma, S. Y., Emborg, M., Bakay, R., Deglon, N., Hantraye, P., Aebischer, P., and Kordower, J. H. (2002). Lentivirally delivered glial cell line-derived neurotrophic factor increases the number of striatal dopaminergic neurons in primate models of nigrostriatal degeneration. J. Neurosci. 22, 4942-4954.

Prasad, K., and Richfield, E. K. (2010). Number and nuclear morphology of $\mathrm{TH}+$ and $\mathrm{TH}-$ neurons in the mouse ventral midbrain using epifluorescence stereology. Exp. Neurol. 225, 328-340.

Ragsdale, C. W., and Graybiel, A. M. (1981). The fronto-striatal projection in the cat and monkey and its relationship to inhomogeneities established by acetylcholinesterase 
histochemistry. Brain Res. 208, 259-266.

Redies, C., Kovjanic, D., Heyers, D., Medina, L., Hirano, S., Suzuki, S. T., and Puelles, L. (2002). Patch/matrix patterns of gray matter differentiation in telencephalon of chicken and mouse. Brain Res. Bull. 57, 489-493.

Rivera, A., Cuellar, B., Giron, F. J., Grandy, D. K., de la Calle, A., and Moratalla, R. (2002). Dopamine D4 receptors are heterogeneously distributed in the striosomes/matrix compartments of the striatum. J. Neurochem. 80, 219-229.

Rodriguez, M., Morales, I., Gomez, I., Gonzalez, S., Gonzalez-Hernandez, T., and Gonzalez-Mora, J. L. (2006). Heterogeneous dopamine neurochemistry in the striatum: the fountain-drain matrix. J. Pharmacol. Exp. Ther. 19, 31-43.

Rymar, V.V., Sasseville, R., Luk, K. C., and Sadikot,A.F. (2004). Neurogenesis and stereological morphometry of calretinin immunoreactive GABAergic interneurons of the neostriatum. $J$. Comp. Neurol. 469, 325-339.

Sadikot, A. F., and Sasseville, R. (1997). Neurogenesis in the mammalian neostriatum and nucleus accumbens: parvalbumin-immunoreactive GABAergic interneurons. J. Comp. Neurol. 389, 193-211.

Saka, E., Iadarola, M., Fitzgerald, D. J., and Graybiel, A. M. (2002). Local circuit neurons in the striatum regulate neural and behavioral responses to dopaminergic stimulation. Proc. Natl. Acad. Sci. U.S.A. 99, 9004-9009.

Sako, W., Morigaki, R., Nagahiro, S., Kaji, R., and Goto, S. (2010). Olfactory type $G$ protein alpha subunit in striosomematrix dopamine systems in adult mice. Neuroscience 170, 497-502.

Sato, K., Sumi-Ichinose, C., Kaji, R., Ikemoto, K., Nomura, T., Nagatsu, I., Ichinose, H., Ito, M., Sako, W., Nagahiro, S., Graybiel, A. M., and Goto, S. (2008). Differential involvement of striosome and matrix dopamine systems in a transgenic model of dopa-responsive dystonia. Proc. Natl. Acad. Sci. U.S.A. 105, 12551-12556.

Schatzmiller, R. A., Goldman, J. S., Simard-Emond, L., Rymar, V., Manitt, C., Sadikot, A. F., and Kennedy, T. E. (2008). Graded expression of netrin-1 by specific neuronal subtypes in the adult mammalian striatum. Neuroscience 157, 621-636.

Tande, D., Hoglinger, G., Debeir, T., Freundlieb,N.,Hirsch,E.C., and Francois, C. (2006). New striatal dopamine neurons in MPTP-treated macaques result from a phenotypic shift and not neurogenesis. Brain 129, 1194-1200.

Tashiro, Y., Sugimoto, T., Hattori, T., Uemura, Y., Nagatsu, I., Kikuchi, H., and Mizuno, N. (1989a). Tyrosine hydroxylase-like immunoreactive neurons in the striatum of the rat. Neurosci. Lett. 97, 6-10.
Tashiro, Y., Kaneko, T., Sugimoto, T., Nagatsu, I., Kikuchi, H., and Mizuno, N. (1989b). Striatal neurons with aromatic L-amino acid decarboxylase-like immunoreactivity in the rat. Neurosci. Lett. 100, 29-34.

Tepper, J. M. (2010). "GABAergic interneurons of the striatum," in Handbook of Basal Ganglia Structure and Function, eds H. Steiner and $\mathrm{K}$. Y. Tseng (San Diego, CA: Academic Press), 151-163.

van Vulpen, E. H., and van der Kooy, D. (1998). Striatal cholinergic interneurons: birthdates predict compartmental localization. Brain Res. Dev. Brain Res. 109, 51-58.

Voorn, P., Gerfen, C. R., and Groenewegen, H. J. (1989). Compartmental organization of the ventral striatum of the rat: immunohistochemical distribution of enkephalin, substance P, dopamine and calcium binding protein. $J$. Comp. Neurol. 289, 189-201.

Voorn, P., Vanderschuren, L. E., and Groenewegen, H. J. (2004). Putting a spin on the dorsal-ventral divide of the striatum. Trends Neurosci. 27,468-474

Wightman, R. M., Heien, M. L., Wassum, K. M., Sombers, L. A., Aragona, B. J., Khan, A. S., Ariansen, J. L., Cheer, J. F., Phillips, P.E., and Carelli, R. M. (2007). Dopamine release is heterogeneous within microenvironments of the rat nucleus accumbens. Eur. J. Neurosci. 26, 2046-2054.
Wu, Y., and Parent, A. (2000). Striatal interneurons expressing calretinin, parvalbumin and NADPH-diaphorase: a comparative study in the rat, monkey and human. Brain Res. 863, 182-191.

Zaborszky, L., Alheid, G. F., Beinfeld, M. C., Eiden, L. E., Heimer, L., and Palkovita,M.(1985). Cholecystokinin innervation of the ventral striatum: a morphological and radioimmunological study. Neuroscience 14, 427-453.

Conflict of Interest Statement: The authors declare that the research was conducted in the absence of any commercial or financial relationships that could be construed as a potential conflict of interest.

Received: 10 March 2011; accepted: 24 May 2011; published online: 06 June 2011.

Citation: Ünal B, Ibáñez-Sandoval O, Shah F, Abercrombie ED and Tepper JM (2011) Distribution of tyrosine hydroxylaseexpressing interneurons with respect to anatomical organization of the neostriatum. Front. Syst. Neurosci. 5:41. doi: 10.3389/ fnsys.2011.00041

Copyright (๐) 2011 Ünal, Ibáñez-Sandoval, Shah, Abercrombie and Tepper. This is an open-access article subject to a nonexclusive license between the authors and Frontiers Media SA, which permits use, distribution and reproduction in other forums, provided the original authors and source are credited and other Frontiers conditions are complied with. 